This is a postprint version of the following published document:

Calle, I.A., Ledesma, P., Castronuovo, E.D. (2015). Advanced application of transient stability constrained-optimal power flow to a transmission system including an HVDC-LCC link. IET Generation, Transmission \& Distribution, v. 9 (13), pp. 1765-1772. DOI: $\underline{10.1049 / \text { iet-gtd.2015.0215 }}$

(C) Institution of Engineering and Technology (IET), 2015 


\title{
Advanced application of transient stability constrained-optimal power flow to a transmission system including an HVDC-LCC link
}

\author{
Ignacio A. Calle ${ }^{1 凶}$, Pablo Ledesma ${ }^{2}$, Edgardo D. Castronuovo ${ }^{2}$ \\ ${ }^{1}$ Department of Electrical Engineering, Universidad Técnica Federico Santa María, Av. España 1680, Valparaíso, Chile \\ ${ }^{2}$ Department of Electrical Engineering, Universidad Carlos III de Madrid, Av. de la Universidad 30, 28911 Leganés, Spain \\ $凶$ E-mail: ignacio.calle@usm.cl
}

\begin{abstract}
In this study, a transient stability constrained-optimal power flow (TSC-OPF) model is proposed and applied to a real case in the currently interconnected Balearic Islands-Iberian Peninsula system. The TSC-OPF retains the dynamics of all generators in the islands with fourth degree transient synchronous generator models and includes a representation of a high-voltage direct current (HVDC) link on the inverter side. The proposed large-scale non-linear optimisation problem, programmed in GAMS and solved using the CONOPT tool, is used to assess the economic impact of the HVDC on the Balearic Islands generation cost under different circumstances, using TSC-OPF and traditional OPF on the dispatch. Different recovery patterns of the HVDC link after a severe fault in the transmission grid are evaluated from the viewpoint of the cost and stability of power generation.
\end{abstract}

\section{Nomenclature}

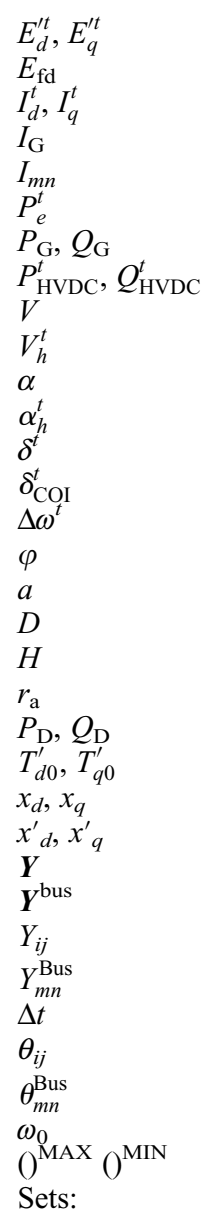

generator internal transient voltage components, p.u. field voltage, p.u.

generator output current components, p.u. magnitude of generator output current, p.u. current between buses $m$ and $n$, p.u. generator active power output, p.u. generator active and reactive power output, p.u. HVDC link active and reactive power output, p.u. bus voltage magnitude, p.u.

voltage at the HVDC connection bus, p.u. bus voltage phase, rad.

voltage phase at the HVDC connection bus, rad. generator angular deviation, rad. centre of inertia angle deviation, rad. generator speed deviation, p.u. bus angle between current and voltage, rad. generation price, M.U/MW damping coefficient, p.u. inertia constant, $s$ armature resistance, p.u. active and reactive power demand, p.u. generator transient time constants, $s$ $d q$-axes synchronous reactance, p.u. $d q$-axes transient reactance, p.u. reduced admittance matrix, p.u. bus admittance matrix, p.u. magnitude of the element $(i, j)$ of $\boldsymbol{Y}$, p.u. magnitude of the element $(m, n)$ of $\boldsymbol{Y}^{\text {bus }}$, p.u. time step, $s$

phase of the element $(i, j)$ of $\boldsymbol{Y}, \operatorname{rad}$. phase of the element $(m, n)$ of $\boldsymbol{Y}^{\text {bus }}, \operatorname{rad}$. frequency reference, $\mathrm{rad} . / s$ upper and lower limits of the variables $\mathcal{G}$, generators; $\mathcal{H} \mathcal{V D C}$,HVDC buses; $\mathcal{L}$, non-generator buses; $\mathcal{N}$, buses; $\mathcal{T}$, time steps.

\section{Introduction}

Transient stability constrained-optimal power flow (TSC-OPF) techniques include an economic objective and static and dynamic constraints in the same optimisation problem [1-3]. Therefore TSC-OPF is an adequate tool for creating a transparent mechanism for calculating optimal economic operation when the system is restricted by steady-state and stability constraints.

During the past decade, TSC-OPF techniques have received increasing attention, with clearly differentiated approaches for representing and assessing the problem of transient stability [1, 4]. In the traditional TSC-OPF methods, transient stability constraints are formulated as rotor angle swing equations [1, 5-13]. The differential equations used in the dynamic models of synchronous machines are converted to algebraic form, using implicit numerical integration methods, such as the trapezoidal rule and are included in the optimisation model [5]. This technique increases the dimensions of the problem because it introduces large numbers of variables and equations that grow with the number of integration steps. Previously, most studies have focused on reducing the size of the problem and improving the computational efficiency. For example, Chen et al. [6] proposed a method based on functional transformation techniques and converted an infinite-dimensional into a finite-dimensional optimisation problem. In [7, 8], a modified formulation using the reduced admittance matrix to represent the electric power in the swing equation was used, which considerably reduced the equality constraints. These studies allowed in [7] to implement multi-contingencies studies. In [9], steady-state and dynamic behaviours were separately analysed using different solvers. In [10], the authors propose a penalty-based approach in which the adjoint equation method is applied to evaluate the gradient of the penalty term associated with the stability constraints, improving the method proposed in [6]. In [11], a method for calculating the Jacobian and Hessian matrices is introduced to reduce the massive calculation of these matrices when functional transformation techniques are used. In [12], considering the truncation error of specific numerical integration 
algorithms, the differential equations are discretised as inequality constraints rather than equality constraints, to improve the computational efficiency. In [13] a hybrid dynamic optimisation approach is proposed for efficient and robust solution of stabilityconstrained optimal power flow problems. This approach combines the algorithmic advantages from direct sequential and simultaneous approaches and it is based on the direct multiple shooting method. All previous studies use the classical dynamic model to represent the generators because it only requires two differential equations for each power plant. This approach reduces the computational burden of the problem at the cost of neglecting electromagnetic transients in the rotor that have an impact on transient stability [14].

Other approaches are based on direct methods (Lyapunov functions; equal area criterion etc.), as in [2, 15-21]. In these studies, generators are classified as critical or non-critical machines and the single-machine equivalent (SIME) method is generally used to obtain the most simplified equivalent system. The angle trajectory on a one-machine infinity-bus (OMIB) equivalent is used to obtain information about the transient stability margins. Direct methods require less computational effort but can introduce non-negligible errors because of the highly non-linear nature of the problem [1].

This paper follows a traditional approach, in which the dynamics of all power plants in the system are retained and the TSC-OPF model is solved as a whole. The resulting model is a large-scale non-linear optimisation problem, programmed in GAMS [22] and solved using the CONOPT tool [23]. The proposed TSC-OPF model is applied to the Balearic Islands power system in Spain. This system covers three islands that are interconnected by submarine AC cables and was recently (2013) connected to the mainland by a high-voltage direct current link with line-commutated converters (HVDC-LCC). This link has an important impact on the economic dispatch, because the cost of energy is lower on the mainland than on the islands. In addition, this link affects other critical technical aspects, such as transient stability. The proposed TSC-OPF model includes two novel features, described in Section 2. First, an HVDC-LCC link is included in the optimisation problem, modelled as a power injection and that can represent different recovery strategies after a fault. Second, the implementation of the transient synchronous two-axes generator model improves the accuracy of the simulation by representing the electromagnetic transients in the stator. In Section 3, the mathematical formulation of the proposed TSC-OPF is explained and in Section 4 the Balearic power system is described.

The TSC-OPF model proposed in this paper is used to analyse several alternatives to recover the HVDC link after a fault based on the viewpoint of economic dispatch and transient stability, in Section 5. From the results, the proposed TSC-OPF is an interesting tool to calculate the optimal strategy for recovering the HVDC link after a severe fault in the islands transmission grid, without significantly affecting the stability of the system. Finally, conclusions are presented in Section 6.

\section{System representation}

The TSC-OPF model contains two different parts [1]. The first part corresponds with pre-fault steady-state conditions and includes all conventional OPF constraints, including power flow equations, the capability limits of generators and voltage, current limits throughout the grid and equality constraints for determining the initial conditions of the dynamic variables. The second part corresponds with the transient stage and includes equality constraints (to represent the dynamic equations of the generators at the fault and post-fault stages) and inequality constraints (to represent the stability condition). Transient equations are formulated by using a reduced admittance matrix, which is obtained by applying the Kron reduction to the original nodal admittance matrix [24]. The reduced admittance matrix usually only retains the internal nodes of the generators. In addition to the generator buses, the point of common coupling (PCC) of the HVDC is also retained in the present formulation. Next subsections explain two novel features included in the proposed TSC-OPF model, the HVDC link and the synchronous generator two-axes transient model.

\section{$2.1 \quad H V D C-L C C$ model for TSC-OPF}

The HVDC link between the continent and the Balearic Islands is a $2 \times 200 \mathrm{MW}$ double link with $\pm 250 \mathrm{kV}$ transmission cables based on LCC technology. Its length is $244 \mathrm{~km}$ with a maximum depth of $1485 \mathrm{~m}$ below sea level, and it is operated under normal conditions at a maximum power of $310 \mathrm{MW}$. During normal operations, the rectifier side of the HVDC functions in current control mode and the inverter side functions in voltage control mode, resulting in constant power injection at the HVDC output bus $[14,25]$. For example, if the voltage decreases slightly in the output bus, the current from the HVDC increases to maintain a constant power. However, the current increase cannot exceed 10 to $20 \%$ of the rated current $[14,26]$.

When a three-phase fault occurs near the inverter side in the AC system, the voltage decreases more or less depending on the location and the severity of the disturbance. The voltage dependent current order limit (VDCOL) function plays an important role during voltage dips because it imposes a limit on the maximum current when the $\mathrm{AC}$ voltage drops below a predetermined value. Fig. $1 a$ shows a typical VDCOL function, such as the one implemented in the studied case [14]. The minimum voltage, $V_{\min }$, is the limit under which the HVDC disconnects and the maximum voltage, $V_{\max }$, is the limit above which the HVDC can operate without restrictions on the current.

Transient stability studies consider worst-case scenarios in which the most severe disturbances are analysed. Preliminary simulations of the relatively small power system considered in this paper and described in Section 4, indicated that a 3-phase short circuit at the transmission level near the HVDC inverter side always results in a
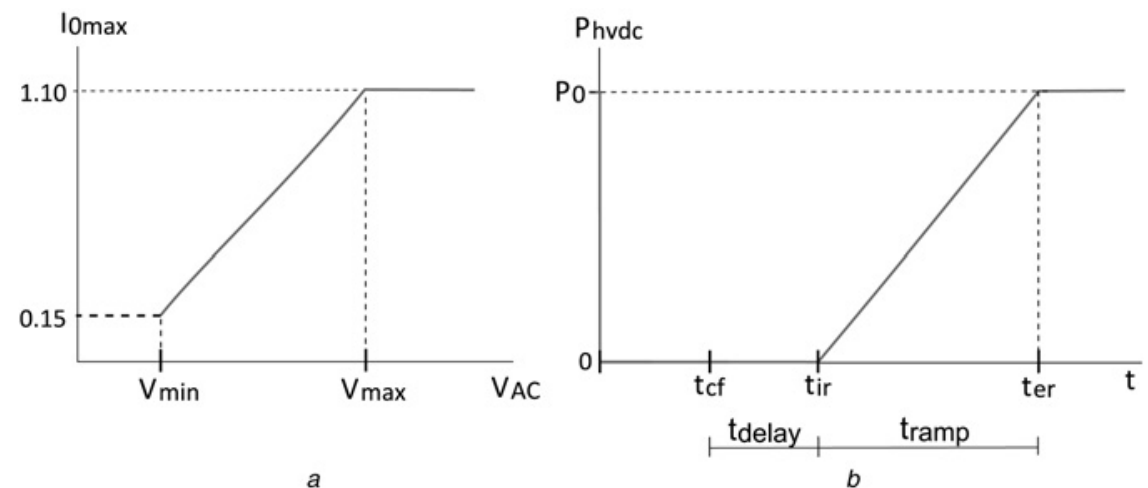

Fig. 1 Dynamic behaviour of the HVDC facing disturbances

$a$ Typical VDCOL function

$b$ Curve of the active power provided by the HVDC link during and after a voltage drop at the PCC 


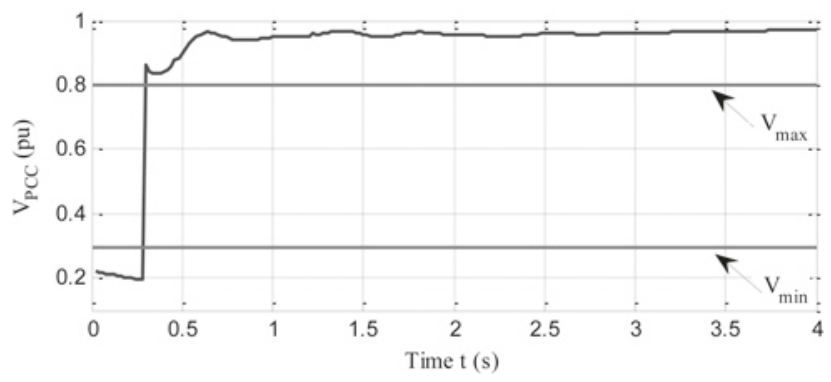

Fig. 2 Voltage profile in the connection bus of the HVDC link

voltage dip under $V_{\min }$ that triggers the disconnection of the HVDC. In addition, once the fault is cleared, the voltage at the PCC of the HVDC recovers to a level greater than $V_{\text {max }}$. Fig. 2 shows an example of the voltage at the PCC of the HVDC during and after a short-circuit in the transmission network.

Fig. $1 b$ shows a typical curve of the active power provided by the HVDC link during and after a severe voltage drop at the PCC. When the fault occurs at time $t=0$, the voltage decreases to below $V_{\min }$ and the output power from the HVDC is decreased to zero. After the fault is cleared at $t=t_{\mathrm{cf}}$, a delay time of $t_{\text {delay }}$ is applied to allow the voltage to stabilise. At $t=t_{i r}=t_{\mathrm{cf}}+t_{\text {delay, }}$, the HVDC begins operating again and the power control gradually increases the power set point during a time $t_{\text {ramp}}$, until the pre-fault value is reached at $t=t_{e r}$. Mathematically, this model is written as follows

$$
P_{\mathrm{HVDC}_{h}}^{t}= \begin{cases}P_{\mathrm{HVDC}_{h}}^{0} & \text { at } t=0 \\ 0 & \text { at } 0<t \leq t_{\mathrm{ir}} \\ P_{\mathrm{HVDC}_{h}}^{0}\left(t-t_{\mathrm{ir}}\right) / t_{\mathrm{ramp}} & \text { at } t_{\mathrm{ir}}<t \leq t_{e r} \\ P_{\mathrm{HVDC}_{h}}^{0} & \text { at } t_{e r}<t\end{cases}
$$

where the power at $t=0$ is calculated using steady-state and dynamic initial equations in the pre-fault stage.

The HVDC-LCC link always demands reactive power during its operation. In the present case, the reactive power demanded by the HVDC-LCC is assumed to be $50 \%$ of the injected active power. The values of $t_{\text {delay }}$ and $t_{\text {ramp }}$ are important because a fast recovery of the HVDC-LCC after a short-circuit can imply that a weak AC system (such as the one studied in this paper) could have problems in providing sufficient reactive power at the rate required by the HVDC. The time for a HVDC system to recover to $90 \%$ of its pre-fault power is typically between 100 and $500 \mathrm{~ms}$, depending on the DC and AC system characteristics and the control strategy used [27]. Different values of $t_{\text {delay }}$ and $t_{\text {ramp }}$, including $t_{\text {ramp }}=0$ (i.e. a step recovery), are evaluated in Section 5 to analyse their impact on operation costs.

The response of the control of an HVDC system to a fault in the AC network is very fast, compared with the time frame of electromechanical oscillations between synchronous generators. This difference in the speed of response makes it possible to neglect the dynamics of the controls of HVDC links in transient stability studies and to model the HVDC link as a power injection. Consequently, the following equations represent the active and reactive power injected by an HVDC-LCC link connected at bus $h$ during the transient stage (see (2))

where $V_{v} \mid \alpha_{v}$ is the voltage at the connection bus of the $v$ th HVDC link, and $E_{d j}^{\prime}, E_{q j}^{\prime}$ and $\delta_{j}$ are the $d$ and $q$ components and the angle of the internal voltage of the $j$ th generator. $Y_{h j} \mid \theta_{h j}$ is the $(h, j)$ element of the reduced admittance matrix that only retains the connection buses of the HVDC links and the internal nodes of the synchronous generators.

\subsection{Transient model of the synchronous generator for TSC-OPF}

Owing to the heavy computational effort required to solve the TSC-OPF model, previous studies retained the dynamics of all power plants by using the classical model to represent synchronous generators $[5,7]$. To improve the accuracy of this study, it is used the fourth order synchronous generator transient model, which makes it possible to represent the electromagnetic transients in the generator rotor. These transients affect the electromagnetic torque of the machines, and the transient stability of the system. The well-known electrical equations of the transient model are $[14,24]$

$$
\left.\begin{array}{c}
\mathrm{d} E_{d i}^{\prime} / \mathrm{d} t=\left(-E_{d i}^{\prime}+\left(x_{q i}-x_{q i}^{\prime}\right) I_{q i}\right) / T_{q 0 i}^{\prime} \\
\mathrm{d} E_{q i}^{\prime} / \mathrm{d} t=\left(-E_{q i}^{\prime}-\left(x_{d i}-x_{d i}^{\prime}\right) I_{d i}+E_{\mathrm{fdi}}\right) / T_{d 0 i}^{\prime} \\
E_{d i}^{\prime}=V_{d i}+r_{\mathrm{a} i} I_{d i}-x_{q i}^{\prime} I_{q i} \\
E_{q i}^{\prime}=V_{q i}+r_{\mathrm{a} i} I_{q i}+x_{d i}^{\prime} I_{d i}
\end{array}\right\}
$$

where $V_{d i}=V_{i} \sin \left(\delta_{i}-\alpha_{i}\right), \quad V_{q i}=V_{i} \cos \left(\delta_{i}-\alpha_{i}\right), I_{d i}=I_{\mathrm{G} i} \sin \left(\delta_{i}-\right.$ $\left.\alpha_{i}+\varphi_{i}\right), I_{q i}=I_{\mathrm{G} i} \cos \left(\delta_{i}-\alpha_{i}+\varphi_{i}\right)$.

The mechanical equations are

$$
\begin{gathered}
\mathrm{d} \Delta \omega_{i} / \mathrm{d} t=\left(P_{m i}-P_{e i}-D_{i} \Delta \omega_{i}\right) /\left(2 H_{i}\right) \\
\mathrm{d} \delta_{i} / \mathrm{d} t=\omega_{0} \Delta \omega_{i} \\
P_{e i}=E_{d i}^{\prime} I_{d i}+E_{q i}^{\prime} I_{q i}
\end{gathered}
$$

The trapezoidal rule is used to discretise differential equations (3)(4) and (6)-(7) and the resulting equations are included in the optimisation model (as shown in Section 3).

All machine electrical variables are referred to a $d q$ reference frame fixed to the rotor of the generator. Since grid electrical variables are referred to in a common synchronous rotating $\alpha \beta$ reference frame, an additional $d q-\alpha \beta$ rotation is required at each machine to calculate the voltage or current within the grid. To reduce the number of constraints in the optimisation model, electrical variables at the grid are eliminated and the current $I_{d i}+$ $j I_{q i}$, is calculated as a function of the internal voltages [24]

$$
\left.\begin{array}{l}
I_{d i}=\sum_{\forall j} Y_{i j}\left(E_{d j}^{\prime} \cos \left(\delta_{i}-\delta_{j}-\theta_{i j}\right)+E_{q j}^{\prime} \sin \left(\delta_{i}-\delta_{j}-\theta_{i j}\right)\right) \\
I_{q i}=\sum_{\forall j} Y_{i j}\left(E_{q j}^{\prime} \cos \left(\delta_{i}-\delta_{j}-\theta_{i j}\right)-E_{d j}^{\prime} \sin \left(\delta_{i}-\delta_{j}-\theta_{i j}\right)\right)
\end{array}\right\}
$$

\section{Mathematical formulation}

This section provides the complete set of equations that constitute the proposed TSC-OPF model, to facilitate the replication of the results. Since the Balearic power system is not currently operated as a liberalised market, the objective function includes the production costs of the power plants. In this application, the cost is computed as a linear function of power plants production. The cost of the power injected from the HVDC link depends on the Iberian market. A linear representation of the prices in the Iberian day-ahead market is included in the simulations obtained from data provided by the Iberian Market Operator [28]. From the economic viewpoint, the HVDC injection is dispatched as any

$$
\left.\begin{array}{rl}
P_{H V D C_{h}} & =V_{h} \sum_{\forall v} V_{v} Y_{h v} \cos \left(\alpha_{h}-\alpha_{v}-\theta_{h v}\right)-V_{h} \sum_{\forall j} Y_{h j}\left(E_{d j}^{\prime} \sin \left(\alpha_{h}-\delta_{j}-\theta_{h j}\right)-E_{q j}^{\prime} \cos \left(\alpha_{h}-\delta_{j}-\theta_{h j}\right)\right) \\
Q_{H V D C_{h}} & =V_{h} \sum_{\forall v} V_{v} Y_{h v} \sin \left(\alpha_{h}-\alpha_{v}-\theta_{h v}\right)+V_{h} \sum_{\forall j} Y_{h j}\left(E_{d j}^{\prime} \cos \left(\alpha_{h}-\delta_{j}-\theta_{h j}\right)+E_{q j}^{\prime} \sin \left(\alpha_{h}-\delta_{j}-\theta_{h j}\right)\right)
\end{array}\right\}
$$


other conventional producer on the Islands. The complete mathematical formulation of the considered TSC-OPF model is

$$
\text { MIN. } f\left(P_{\mathrm{G} i}, P_{\mathrm{HVDC}_{h}}^{0}\right)=\sum\left(a_{i} P_{\mathrm{G} i}+a_{h} P_{\mathrm{HVDC}_{h}}^{0}\right)
$$

subject to

$$
\left.\begin{array}{r}
P_{\mathrm{G} i}-P_{\mathrm{D} i}-V_{i} \sum_{\forall n} V_{n} Y_{i n}^{\mathrm{Bus}} \cos \left(\alpha_{i}-\alpha_{n}-\theta_{i n}^{\mathrm{Bus}}\right)=0 \\
P_{\mathrm{HVDC}_{h}}^{0}-P_{\mathrm{D} h}-V_{h} \sum_{\forall n} V_{n} Y_{h n}^{\mathrm{Bus}} \cos \left(\alpha_{h}-\alpha_{n}-\theta_{h n}^{\mathrm{Bus}}\right)=0 \\
-P_{\mathrm{D} l}-V_{l} \sum_{\forall n} V_{n} Y_{l n}^{\mathrm{Bus}} \cos \left(\alpha_{l}-\alpha_{n}-\theta_{l n}^{\mathrm{Bus}}\right)=0
\end{array}\right\}
$$

(see (16))

$$
\left.\begin{array}{c}
I_{d i}^{0}-I_{\mathrm{G} i} \sin \left(\delta_{i}^{0}-\alpha_{i}+\varphi_{i}\right)=0 \\
I_{q i}^{0}-I_{\mathrm{G} i} \cos \left(\delta_{i}^{0}-\alpha_{i}+\varphi_{i}\right)=0
\end{array}\right\}
$$

(see (19) and (20))

$$
P_{e i}^{t}-E_{d i}^{\prime t} I_{d i}^{t}-E_{q i}^{\prime t} I_{q i}^{t}=0
$$

(see (22))

$$
Q_{\mathrm{HVDC}_{h}}^{t}+P_{\mathrm{HVDC}_{h}}^{t} / 2=0
$$

$$
\left.\begin{array}{lll}
P_{\mathrm{HVDC}_{h}}^{t}=0 & \text { at } & 0<t \leq t_{\mathrm{ir}} \\
P_{\mathrm{HVDC}_{h}}^{t}-P_{\mathrm{HVDC}_{h}}^{0}\left(t-t_{\mathrm{ir}}\right) / t_{\mathrm{ramp}}=0 & \text { at } & t_{\mathrm{ir}}<t \leq t_{\text {er }} \\
P_{\mathrm{HVDC}_{h}}^{t}-P_{\mathrm{HVDC}_{h}}^{0}=0 & \text { at } \quad t_{e r}<t
\end{array}\right\}
$$

$($ see $(25))$

$$
\begin{gathered}
\delta_{\mathrm{COI}}^{t}-\sum_{i} H_{i} \delta_{i}^{t} / \sum_{i} H_{i}=0 \\
-\delta_{\mathrm{Stab} .}^{\mathrm{MAX} .} \leq \delta_{i}^{t}-\delta_{\mathrm{COI}}^{t} \leq \delta_{\mathrm{Stab} .}^{\mathrm{MAX} .} \\
V_{m}^{\mathrm{MIN} .} \leq V_{m} \leq V_{m}^{\mathrm{MAX} .} ; \quad \alpha_{m}^{\mathrm{MIN} .} \leq \alpha_{m} \leq \alpha_{m}^{\mathrm{MAX} .} ; \\
0 \leq I_{m n} \leq I_{m n}^{\mathrm{MAX} .} \\
P_{\mathrm{G} i}^{\mathrm{MIN} .} \leq P_{\mathrm{G} i} \leq P_{\mathrm{G} i}^{\mathrm{MAX} .} ; \quad 0 \leq I_{\mathrm{G} i} \leq I_{\mathrm{G} i}^{\mathrm{MAX} .} ; \\
P_{\mathrm{G} i}^{2}+\left(Q_{\mathrm{G} i}+V_{\mathrm{G} i}^{2} / x_{\mathrm{D} i}^{\prime}\right)^{2} \leq\left(E_{\mathrm{MAX}} V_{\mathrm{G} i} / x^{\prime}{ }^{\prime}\right)^{2} \\
\Delta \omega^{\mathrm{MIN} .} \leq \Delta \omega_{i}^{t} \leq \Delta \omega^{\mathrm{MAX} .} ; \quad \delta^{\mathrm{MIN} .} \leq \delta_{i}^{t} \leq \delta^{\mathrm{MAX} .} \\
E_{d i}^{\prime \mathrm{MIN} .} \leq E_{d i}^{\prime t} \leq E_{d i}^{\prime \mathrm{MAX} .} ; \quad E_{q i}^{\mathrm{MIN} .} \leq E_{q i}^{\prime t} \leq E_{q i}^{\mathrm{MAX} .} ; \\
E_{\mathrm{fd} i}^{\mathrm{MIN} .} \leq E_{\mathrm{fd} i} \leq E_{\mathrm{fd} i}^{\mathrm{MAX} .} ; \quad I_{d i}^{\mathrm{MIN} .} \leq I_{d i}^{t} \leq I_{d i}^{\mathrm{MAX} .} ; \quad I_{q i}^{\mathrm{MIN} .} \leq I_{q i}^{t} \leq I_{q i}^{\mathrm{MAX} .}
\end{gathered}
$$

$\forall h, v \in\{\mathcal{H} \mathcal{V D C}\} ; \quad \forall i, j \in\{\mathcal{G}\} ;$

$\forall l \in\{\mathcal{L}\} ; \quad \forall m, n \in\{\mathcal{N}\} ; \quad \forall t \in\{\mathcal{T}\}$

The meaning of the constraints is described below.

- Equations (11) and (12) represent the active and reactive power balances in the generation, non-generation and HVDC buses. Bus 1 is the slack bus, with a fixed angle of $\alpha_{1}=0$.

- Equations (13) and (14) are used to calculate the currents through the branches of the system and in the generators, respectively.

- Equations (15) to (18) initialise the transient-state variables of the generators $\left(E_{d i}^{\prime 0}, E_{q i}^{\prime 0}, \delta_{i}^{0}, \Delta \omega_{i}^{0}, P_{e i}^{0}, I_{d i}^{0}, I_{q i}^{0}\right)$.

- Equations (19) and (20) result from the application of the trapezoidal rule to the transient stability model of the generators at each time step. The time step used during the fault and post-fault periods is $\Delta t=0.02 \mathrm{~s}$.

$$
\left.\begin{array}{r}
V_{i} \sin \left(\delta_{i}^{0}-\alpha_{i}\right)-E_{d i}^{\prime 0}+\left(r_{\mathrm{a} i} \sin \left(\delta_{i}^{0}-\alpha_{i}+\varphi_{i}\right)-x_{q i}^{\prime} \cos \left(\delta_{i}^{0}-\alpha_{i}+\varphi_{i}\right)\right) I_{\mathrm{G} i}=0 \\
V_{i} \cos \left(\delta_{i}^{0}-\alpha_{i}\right)-E_{q i}^{\prime 0}+\left(r_{\mathrm{a} i} \cos \left(\delta_{i}^{0}-\alpha_{i}+\varphi_{i}\right)+x_{d i}^{\prime} \sin \left(\delta_{i}^{0}-\alpha_{i}+\varphi_{i}\right)\right) I_{\mathrm{G} i}=0
\end{array}\right\}
$$

$$
\begin{aligned}
& E_{d i}^{\prime t+1}\left(1+\Delta t / 2 T_{q 0 i}^{\prime}\right)-E_{d i}^{\prime t}\left(1-\Delta t / 2 T_{q 0 i}^{\prime}\right)-\left(\Delta t / 2 T_{q 0 i}^{\prime}\right)\left(x_{q i}-x_{q i}^{\prime}\right)\left(I_{q i}^{t+1}+I_{q i}^{t}\right)=0 \\
& \left.E_{q i}^{\prime t+1}\left(1+\Delta t / 2 T_{d 0 i}^{\prime}\right)-E_{q i}^{\prime t}\left(1-\Delta t / 2 T_{d 0 i}^{\prime}\right)-\left(\Delta t / 2 T_{d 0 i}^{\prime}\right)\left[2 E_{\mathrm{fd} i}-\left(x_{d i}-x_{d i}^{\prime}\right)\left(I_{d i}^{t+1}+I_{d i}^{t}\right)\right]=0\right\} \\
& \left.\begin{array}{rl}
\Delta \omega_{i}^{t+1}\left(1+D_{i} \Delta t / 4 H_{i}\right)-\Delta \omega_{i}^{t}\left(1-D_{i} \Delta t / 4 H_{i}\right)-\left(\Delta t / 4 H_{i}\right)\left(2 P_{m i}-P_{e i}^{t+1}-P_{e i}^{t}\right) & =0 \\
\delta_{i}^{t+1}-\delta_{i}^{t}-(\Delta t / 2) \omega_{0}\left(\Delta \omega_{i}^{t+1}+\Delta \omega_{i}^{t}\right) & =0
\end{array}\right\}
\end{aligned}
$$

$$
\left.\begin{array}{l}
P_{H V D C_{h}}^{t}-V_{h} \sum_{\forall v} V_{v} Y_{h v} \cos \left(\alpha_{h}-\alpha_{v}-\theta_{h v}\right)+V_{h} \sum_{\forall j} Y_{h j}\left(E_{d j}^{\prime} \sin \left(\alpha_{h}-\delta_{j}-\theta_{h j}\right)-E_{q j}^{\prime} \cos \left(\alpha_{h}-\delta_{j}-\theta_{h j}\right)\right)=0 \\
Q_{H V D C_{h}}^{t}-V_{h} \sum_{\forall v} V_{v} Y_{h v} \sin \left(\alpha_{h}-\alpha_{v}-\theta_{h v}\right)-V_{h} \sum_{\forall j} Y_{h j}\left(E_{d j}^{\prime} \cos \left(\alpha_{h}-\delta_{j}-\theta_{h j}\right)+E_{q j}^{\prime} \sin \left(\alpha_{h}-\delta_{j}-\theta_{h j}\right)\right)=0
\end{array}\right\}
$$

$$
\left.\begin{array}{l}
I_{d_{i}}^{t}-\sum_{\forall j} Y_{i j}\left(E_{d_{j}}^{\prime t} \cos \left(\delta_{i}^{t}-\delta_{j}^{t}-\theta_{i j}\right)+E_{q_{j}}^{\prime t} \sin \left(\delta_{i}^{t}-\delta_{j}^{t}-\theta_{i j}\right)\right)-\sum_{\forall h} Y_{i h} V_{h}^{t} \sin \left(\delta_{i}^{t}-\alpha_{h}^{t}-\theta_{i h}\right)=0 \\
I_{q_{i}}^{t}-\sum_{\forall j} Y_{i j}\left(E_{q_{j}}^{\prime t} \cos \left(\delta_{i}^{t}-\delta_{j}^{t}-\theta_{i j}\right)-E_{d_{j}}^{\prime t} \sin \left(\delta_{i}^{t}-\delta_{j}^{t}-\theta_{i j}\right)\right)-\sum_{\forall h} Y_{i h} V_{h}^{t} \cos \left(\delta_{i}^{t}-\alpha_{h}^{t}-\theta_{i h}\right)=0
\end{array}\right\}
$$




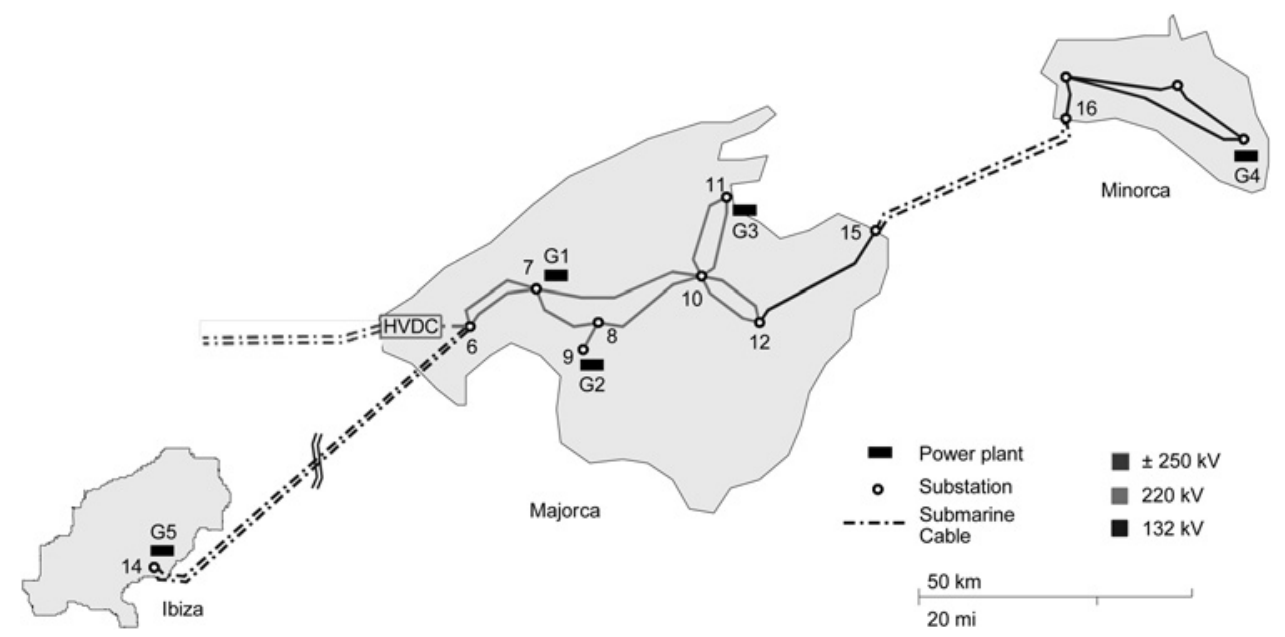

Fig. 3 Map of the power system of Balearic Islands

- Equation (21) calculates the active power output of each generator at each time step as a function of the current and internal voltage.

- Equations (22)-(24) represent the HVDC link during the transient stage. Equations (22) are used to calculate the power inputs/outputs from the HVDC. In (23), the reactive power absorption of the HVDC-LCC link is $50 \%$ of the active power injection. The active power injection is calculated in (24), according to (1).

- Equations (25) are used to calculate the synchronous generator output currents. These equations are a generalisation of (9) and include the HVDC link. The values of these currents are computed as a function of the internal voltages of the machines, the voltage at the PCC of the HVDC link and the reduced admittance matrix.

- Equation (26) is used to calculate the angle of the centre of inertia (COI) at each time step and (27) represents the transient stability criterion $[8,12,29,30]$. The COI provides a reference, making it possible to separate the rotor angle deviations because of the electromechanical oscillations from the deviations that result from the acceleration of the system. Therefore the system is considered fully stable when the separations between any one of the generator angles and the COI remain enclosed in a range.
- Equations (28) represent the limits of the bus voltages and branch currents.

- Equations (29)-(31) specify the range of the rest of variables.

\section{Studied case}

The optimisation problem is applied to the power system of Balearic Islands, in the western Mediterranean Sea near the eastern coast of the Iberian Peninsula. Fig. 3 shows a simplified map of the power system, including the main power plants, the 220 and $132 \mathrm{kV}$ grids, the HVDC submarine cable linking the Iberian Peninsula and Majorca and the $\mathrm{AC}$ submarine cables between the islands. A new $\mathrm{AC}$ submarine cable is being constructed between Majorca and Ibiza Islands and is scheduled to being operating in 2015. In the present case, this cable is also represented using design specifications.

Fig. 4 shows the one-line diagram of the system, which contains five power plants. Here $G_{1}$ and $G_{2}$ are combined cycle power plants with $660 \mathrm{MVA}$ (600 MW) and 520 MVA (470 MW), respectively, $G_{3}$ is a $560 \mathrm{MVA}(510 \mathrm{MW})$ coal-fired power plant

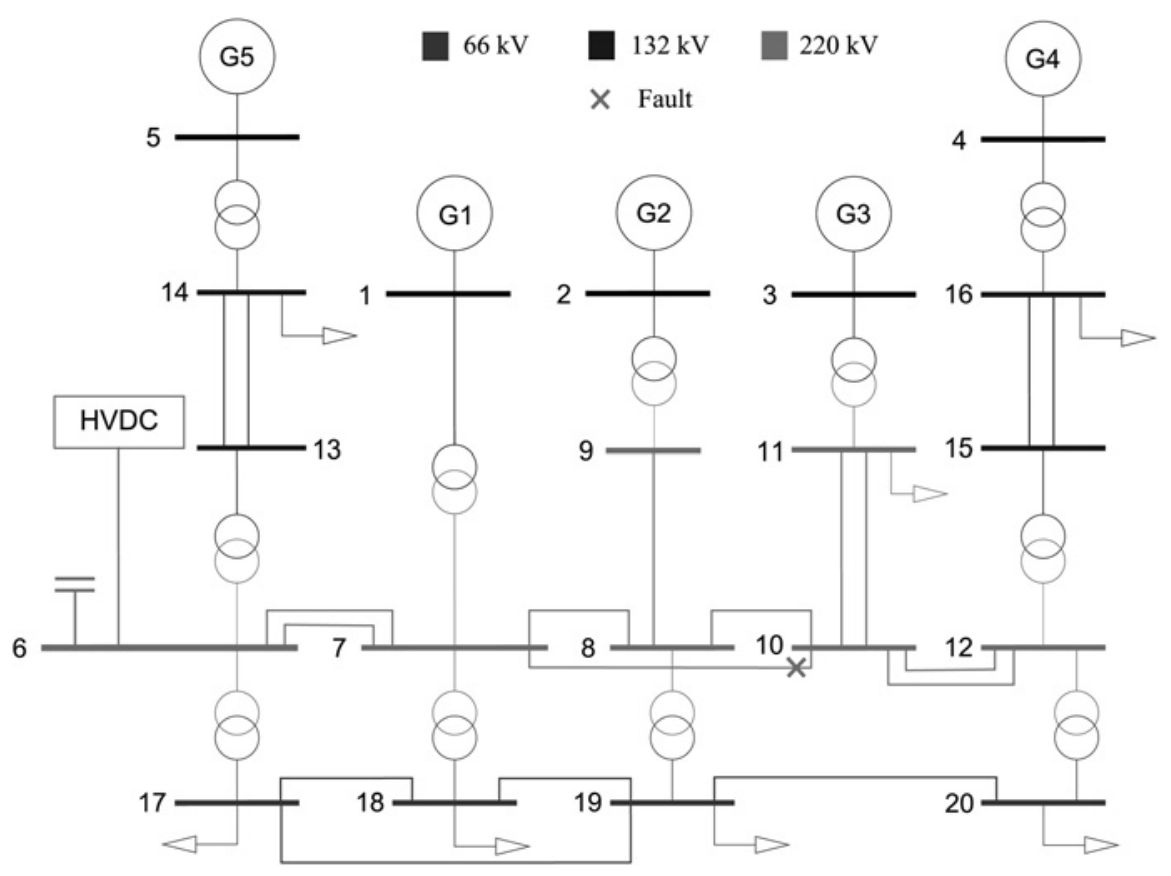

Fig. 4 One-line diagram of the studied case 
and $G_{4}$ and $G_{5}$ are 300 MVA (275 MW) and 385 MVA (350 MW) gas turbine plants, respectively. Bus 6 is the PCC of the submarine HVDC-LCC link connecting the Peninsular and the Balearic power systems. This bipolar link has a rated power of $400 \mathrm{MW}$ with an operating power of $310 \mathrm{MW}$.

The AC transmission grid contains three voltage levels: 220, 132 and $66 \mathrm{kV}$. The HVDC-LCC link is connected to the AC $220 \mathrm{kV}$ level at bus 6 . The $66 \mathrm{kV}$ grid has been included in the model because it is a meshed network that provides alternative routes between the $220 \mathrm{kV}$ buses and affects the power flows during the electromechanical transients after the fault.

The Appendix provides detailed data regarding the dynamic parameters of the generators (Table 4) and the generation prices (Table 5). Parameters of the lines, the transformers, the loads and the limits of operation, can be obtained from [8].

The effect of a three-phase fault to ground in the line between buses 7 and 10 and near to bus 10 is analysed. The fault is cleared after $t_{\mathrm{cf}}=$ $300 \mathrm{~ms}$ by disconnecting the faulty line at both ends. Previous simulations show that this is the most critical disturbance regarding transient stability because it is located near to generator $G_{3}$. Generator $G_{3}$ is one of the largest generators in the system and is generally highly dispatched because it is less expensive than other generators.

\section{Results and discussion}

For comparison purposes, Table 1 shows the generation costs for the studied case from a conventional OPF (only (10)-(14) and (28)-(29) of the model) and from the TSC-OPF ((10)-(31)) when the HVDC link is in operation and when it is not in operation $\left(P_{\mathrm{HVDC}}=Q_{\mathrm{HVDC}}\right.$ $=0$ ). The off-peak load is assumed to be $50 \%$ of the peak load. As shown in the first two columns, when the transient stability is not accounted for, the inclusion of the HVDC link reduces the cost for the peak and off-peak cases by $7.14 \%$ and $12.72 \%$, respectively. The savings provided by the interconnection are higher at peak load $(626.40-581.70=44.70$ M.U.) than at off-peak load $(233.85-$ $204.10=29.75$ M.U.), because at peak load the insular generation displaced by the continental market through the HVDC link is more expensive.

By comparing the first and fourth columns in Table 1, it can be observed that including the transient stability constraints results in generation costs increases of 14 and $54 \%$ for peak and off-peak load scenarios, respectively. The greater cost for providing a stable dispatch results from transferring the power generation from $G_{3}$ to more expensive power plants within the insular system.

\subsection{TSC-OPF with the HVDC link in operation}

When the HVDC link is in operation, the stability of the system is affected by its recovery strategy after the fault. In order to provide examples of the solution of the TSC-OPF, this section shows the results with two extreme cases (as shown in Fig. 1b) a fast recovery $\left(t_{\text {delay }}=0 \mathrm{~ms}, t_{\text {ramp }}=0 \mathrm{~s}\right)$ and a slow recovery $\left(t_{\text {delay }}=\right.$ $300 \mathrm{~ms}, t_{\mathrm{ramp}}=1.5 \mathrm{~s}$ ). In all of the cases studied in this paper, it is used a simulation time of $t_{\max }=4.0 \mathrm{~s}$, a time step of $\Delta t=0.02 \mathrm{~s}$ and the maximum deviation of the rotor angles with respect to the $\mathrm{COI}$ is set at $60^{\circ}$. Each solution is obtained within approximately $110 \mathrm{~s}$, by using a conventional computer with a $3.4 \mathrm{GHz}$ processor and 4 GB RAM.

Fig. $5 a$ shows the rotor angles of the generators provided by the optimal TSC-OPF solution when the system is at peak load and with a fast reconnection of the HVDC link after the fault $\left(t_{\text {delay }}=\right.$ $0 \mathrm{~ms}, t_{\text {ramp }}=0 \mathrm{~s}$ ). The upper and lower limits represent the stability

Table 1 Comparison of generation cost with and without HVDC, in monetary units [m.u.]

\begin{tabular}{lccccc}
\hline OPF - no & $\begin{array}{c}\text { OPF with } \\
\text { HVDC, M.U. }\end{array}$ & $\begin{array}{c}\text { Increase, } \\
\%\end{array}$ & $\begin{array}{c}\text { TSC-OPF - no } \\
\text { HVDC, M.U. }\end{array}$ & $\begin{array}{c}\text { Increase, } \\
\%\end{array}$ \\
\hline $\begin{array}{l}\text { peak } \\
\text { off-peak }\end{array}$ & 626.40 & 581.70 & -7.14 & 716.30 & 14.35 \\
\hline
\end{tabular}
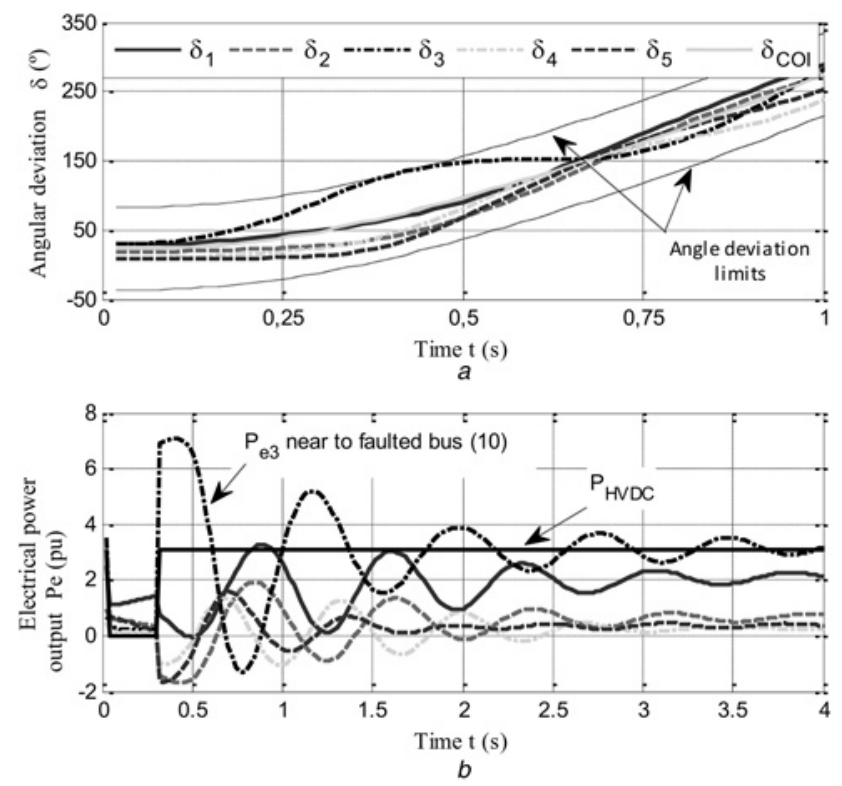

Fig. 5 Fast recovery strategy on the HVDC link

$a$ Rotor angular deviation

$b$ Active power output

margin of $60^{\circ}$ with respect to the COI. The largest deviation occurs at approximately $t=400 \mathrm{~ms}$, when generator $G_{3}$ reaches the angle limit. Fig. $5 b$ shows the active power output from the generators and the HVDC link in the same case and shows that the maximum oscillation corresponds to $G_{3}$. The damping effect of the electromagnetic transients in the rotor progressively reduces the amplitudes of the deviations in the post-fault stage.

Figs. $6 a$ and $b$ show the same variables when a slow reconnection of the HVDC link is applied $\left(t_{\text {delay }}=300 \mathrm{~ms}, t_{\text {ramp }}=1.5 \mathrm{~s}\right)$. Again, the optimal solution occurs when generator $G_{3}$ reaches the angle limit at approximately $t=400 \mathrm{~ms}$. The speed gained by the system after the fault is lower because of the slow recovery of the HVDC link, which is observed when comparing the final values of the COI in Figs. $5 a$ and $6 a$. The recovery pattern of the HVDC following a ramp is shown in Fig. $6 b$, and can be compared with the fast recovery of the HVDC shown in Fig. $5 b$.
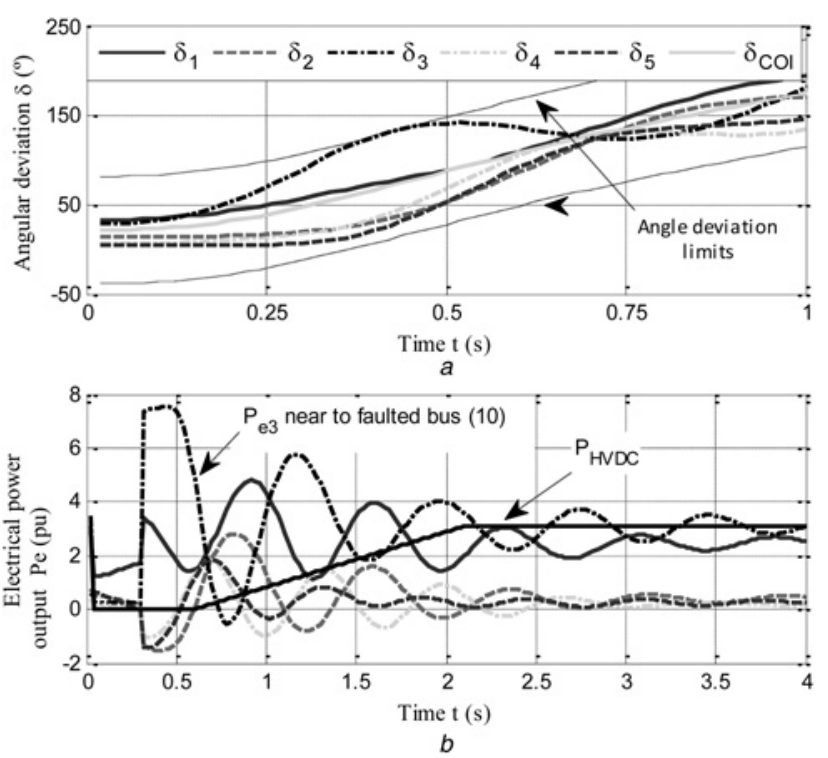

Fig. 6 Slow recovery strategy on the HVDC link

$a$ Rotor angular deviation

$b$ Active power output 
Table 2 Comparison of generation dispatches at peak load

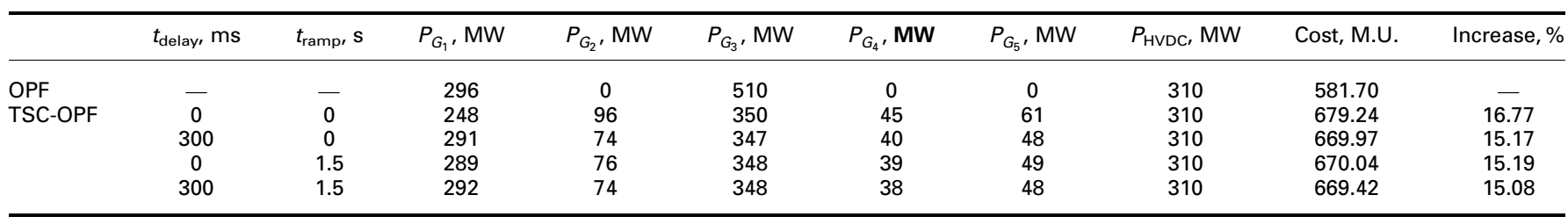

\subsection{Effect of the strategy of recovery of the HVDC on the cost of generation}

To determine the most economic option, the proposed TSC-OPF was systematically applied to different power recovery patterns of the HVDC link. Several values were assigned to parameters $t_{\text {delay }}$ and $t_{\text {ramp }}$ in the peak and off-peak load scenarios.

Table 2 shows the results obtained at peak load for the following extreme values: $t_{\text {delay }}=0$ or $300 \mathrm{~ms}$ and $t_{\text {ramp }}=0$ or $1.5 \mathrm{~s}$. Columns $P_{G_{1}}$ to $P_{G_{5}}$ show the generation assigned to each power plant, column $P_{\mathrm{HVDC}}$ shows the optimal power transmission assigned to the HVDC link, and the Cost column shows the total generation cost in Monetary Units. The last column shows the increasing cost of the corresponding TSC-OPF over the cost of the traditional OPF, which can be considered as the cost of ensuring transient stability when the HVDC link is in operation.

Table 2 shows that the HVDC link is injecting power at its maximum capacity of $310 \mathrm{MW}$ in all five cases. However, to ensure transient stability, generation is transferred from $G_{1}$ and $G_{3}$ to $G_{2}, G_{4}$ and $G_{5}$, which are more expensive power plants. Considering the most economical solution in Table 2, (last row, which is a cost of 669.42 M.U.) and comparing it with the values in Table 1, it can be seen that the cost of ensuring transient stability without HVDC $(716.30-626.40=89.90$ M.U. $)$ is very similar to the case with HVDC $(669.42-581.70=87.72$ M.U. $)$.

Fig. 7 shows the increment in cost of the TSC-OPF relative to the OPF at peak load for several $t_{\text {delay }}$ and $t_{\text {ramp }}$ values, including intermediate values relative to those shown in Table 2. In this case, the cost increases when the HVDC link recovers its pre-fault operation point soon after fault clearance. From Table 2 and Fig. 7, the difference between the recovery strategies at peak load periods is small, $1.7 \%$ at most. However, Fig. 7 indicates that transient stability can be ensured at a slightly lower cost if the recovery strategy for

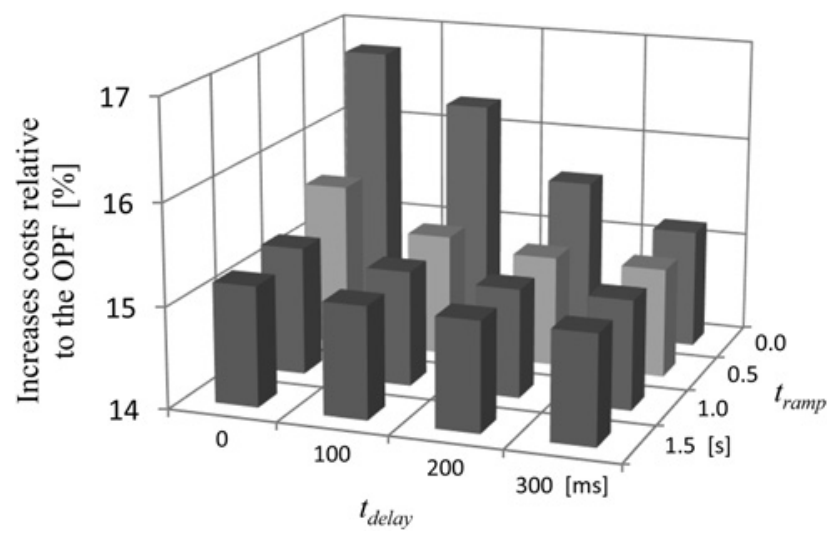

Fig. 7 Increases in the TSC-OPF costs relative to the OPF at peak load the HVDC link combines a certain delay and/or a progressive increase in the active power set point. Moreover, this effect also involves a saturation point, where further cost reductions cannot be obtained even if the delay increases or the power injection ramp slows.

Table 3 and Fig. 8 show the same study conducted on the off-peak scenario. The first row in Table 3 shows that a conventional OPF dispatches the full power from the continental market through the HVDC link, and generator $G_{3}$ to cover the demand. The rows corresponding to the TSC-OPF show that the most economical solution also dispatches the HVDC link to its maximum capacity when transient stability constraints are included.

Fig. 8 shows that it is possible to reduce the generation costs while ensuring transient stability if the recovery of the power injected by the HVDC link is delayed after the fault clearance. This characteristic is similar to that obtained in a peak load scenario, and more acute in terms of relative increments. In this last case, the difference between fast (254.92 M.U.) and slow (231.09 M.U.) recovery strategies is $9.35 \%$.

It is interesting to note that without the HVDC link, the cost for ensuring transient stability is $360.28-233.85=126.43 \mathrm{M} . \mathrm{U}$. (Table 1). When the HVDC link is in operation, the cost for ensuring transient stability is $231.09-204.10=26.99$ M.U. (Table 3). Therefore during off-peak operation, the HVDC link has a positive economic impact not only because it has a lower cost compared with the power plants within the island, but because it reduces the cost to stabilise the power system.

Figs. 7 and 8 can be used to select the recovery strategy of the HVDC link after a short-circuit in the grid. Given that from the point of view of the frequency stability it is interesting to recover the generation as soon as possible, parameters $t_{\text {delay }}=200 \mathrm{~ms}$ and $t_{\text {ramp }}=1.0 \mathrm{~s}$ are a sensible option that results in a significant reduction of costs with respect to a fast reconnection, mainly during off-peak periods.

Table 3 Comparison of generation dispatches at off-peak load

\begin{tabular}{|c|c|c|c|c|c|c|c|c|c|c|}
\hline & $t_{\text {delay, }}, \mathrm{ms}$ & $t_{\text {ramp }}, \mathrm{s}$ & $P_{G_{1}}, \mathrm{MW}$ & $P_{G_{2}}, \mathrm{MW}$ & $P_{G_{3}}, \mathrm{MW}$ & $P_{G_{4}}, \mathrm{MW}$ & $P_{G_{5}}, \mathrm{MW}$ & $P_{\mathrm{HVDC}}, \mathrm{MW}$ & Cost, M.U. & Increase, \% \\
\hline OPF & - & - & 0 & 0 & 239 & 0 & 0 & 310 & 204.10 & --- \\
\hline TSC-OPF & 0 & 0 & 44 & 27 & 133 & 14 & 23 & 310 & 254.92 & 24.90 \\
\hline & 0 & 1.5 & 21 & 16 & 181 & 7 & 15 & 310 & 233.11 & 14.21 \\
\hline & 300 & 1.5 & 18 & 14 & 186 & 7 & 15 & 310 & 231.09 & 13.22 \\
\hline
\end{tabular}




\section{Conclusions}

An improved TSC-OPF model is proposed, including an HVDC-LCC link model with a control of power injection and a transient 4th order synchronous generator model on the $d q$-axes to represent the synchronous machines. The dynamics of all synchronous generators are explicitly retained. The proposed model is applied to a real test case and solved using GAMS. No convergence problems have been observed in the solution of the proposed model.

A practical application of the proposed model is analysed through the study, showing the economic implications of connecting the HVDC-LCC link to an isolated cost-dispatched system. The proposed TSC-OPF model makes it possible to include transient stability constraints in the economic dispatch. It is shown that the stability constraints can increase the generation cost in the studied system between 13 and 54\% (at off-peak scenario, with and without HVDC, respectively), which justifies the use of TSC-OPF over traditional OPF, and the analysis of the HVDC-LCC model.

The proposed TSC-OPF model allows the analysis of different HVDC link recovery patterns after a severe fault regarding generation cost. This approach largely simplifies a study that involves generation costs, static and dynamic constraints and power control. It is found that a fast reconnection of the HVDC link after a fault in the transmission network results in higher costs, while a short delay in the reconnection can save up to $9.35 \%$ of the generation cost.

\section{Acknowledgments}

Pablo Ledesma and Edgardo Castronuovo acknowledge the Ministry of Science and Technology of Spain (Project RESMART, 2014/ 00338/001).

\section{References}

1 Xu, Y., Dong, Z.Y., Xu, Z., Zhang, R., Wong, K.P.: 'Power system transient stability-constrained optimal power flow: a comprehensive review'. 2012 IEEE Power and Energy Society General Meeting, 2012, pp. 1-7

2 Tu, X., Dessaint, L.-A., Nguyen-Duc, H.: 'Transient stability constrained optimal power flow using independent dynamic simulation', IET Gener. Transm. Distrib., 2013, 7, (3), pp. 244-253

3 Verma, K., Niazi, K.R.: 'A coherency based generator rescheduling for preventive control of transient stability in power systems', Int. J. Electr. Power Energy Syst., 2013, 45, (1), pp. 10-18

4 Capitanescu, F., Martinez Ramos, J.L., Panciatici, P., et al: 'State-of-the-art, challenges, and future trends in security constrained optimal power flow', Electr. Power Syst. Res., 2011, 81, (8), pp. 1731-1741

5 Gan, D., Thomas, R.J., Zimmerman, R.D.: 'Stability-constrained optimal power flow', IEEE Trans. Power Syst., 2000, 15, (2), pp. 535-540

6 Chen, L., Taka, Y., Okamoto, H., Tanabe, R., Ono, A.: 'Optimal operation solutions of power systems with transient stability constraints', IEEE Trans. Circuits Syst. I Fundam. Theory Appl., 2001, 48, (3), pp. 327-339

7 Yuan, Y., Kubokawa, J., Sasaki, H.: 'A solution of optimal power flow with multicontingency transient stability constraints', IEEE Trans. Power Syst., 2003, 18, (3), pp. 1094-1102

8 Calle, I.A., Castronuovo, E.D., Ledesma, P.: 'Optimal re-dispatch of an isolated system considering transient stability constraints', Int. J. Electr. Power Energy Syst., 2013, 44, (1), pp. 728-735

9 Layden, D., Jeyasurya, B.: 'Integrating security constraints in optimal power flow studies'. IEEE Power Engineering Society General Meeting, 2004., 2004, vol. 1, pp. $125-129$

10 Sun, Y., Xinlin, Y., Wang, H.F.: 'Approach for optimal power flow with transient stability constraints', IEE Proc.-Gener. Transm. Distrib., 2004, 151, (1), pp. $8-18$
11 Xia, Y., Chan, K.W., Liu, M.: 'Direct nonlinear primal-dual interior-point method for transient stability constrained optimal power flow', IEE Proc.-Gener. Transm. Distrib. 2005, 152, (1), pp. 11-16

12 Jiang, Q., Huang, Z.: 'An enhanced numerical discretization method for transient stability constrained optimal power flow', IEEE Trans. Power Syst., 2010, 25, (4), pp. 1790-1797

13 Geng, G., Ajjarapu, V., Jiang, Q.: 'A hybrid dynamic optimization approach for stability constrained optimal power flow', IEEE Trans. Power Syst., 2014, 29, (5), pp. 2138-2149

14 Kundur, P.: 'Power system stability and control' (McGraw-Hill Professional, 1994)

15 Pizano-Martinez, A., Fuerte-Esquivel, C.R., Ruiz-Vega, D.: 'Global transient stability-constrained optimal power flow using SIME sensitivity analysis'. IEEE Power and Energy Society General Meeting, 2010, pp. 1-8

16 Pizano-Martinez, A., Fuerte-Esquivel, C.R., Ruiz-Vega, D.: 'A new practical approach to transient stability-constrained optimal power flow', IEEE Trans. Power Syst., 2011, PP, (99), p. 1

17 Ruiz-Vega, D., Pavella, M. 'A comprehensive approach to transient stability control. I. near optimal preventive control', IEEE Trans. Power Syst., 2003, 18, (4), pp. 1446-1453

18 Zarate-Minano, R., Van Cutsem, T., Milano, F., Conejo, A.J.: 'Securing transient stability using time-domain simulations within an optimal power flow', IEEE Trans. Power Syst., 2010, 25, (1), pp. 243-253

19 Ahmadi, H., Ghasemi, H., Haddadi, A.M., Lesani, H.: 'Two approaches to transient stability-constrained optimal power flow', Int. J. Electr. Power Energy Syst., 2013, 47, pp. 181-192

20 Tu, X., Dessaint, L., Kamwa, I. 'A global approach to transient stability constrained optimal power flow using a machine detailed model', Can. J. Electr. Comput. Eng., 2013, 36, (1), pp. 32-41

21 Tu, X., Dessaint, L.-A., Kamwa, I.: 'Fast approach for transient stability constrained optimal power flow based on dynamic reduction method', IET Gener. Transm. Distrib., 2014, 8, (7), pp. 1293-1305

22 'GAMS Home Page.'. Available at http://www.gams.com/. [Accessed on 04-Jan-2015]

23 'CONOPT Home Page.'. Available at http://www.conopt.com/. [Accessed on 17-Nov-2014

24 Anderson, P.M., Fouad, A.A.: 'Power system control and stability' (Wiley-IEEE Press, Piscataway, NJ, 2002, 2nd edn.)

25 Montilla-DJesus, M., Santos-Martin, D., Arnaltes, S., Castronuovo, E.D.: 'Optima reactive power allocation in an offshore wind farms with LCC-HVdc link connection', Renew. Energy, 2012, 40, (1), pp. 157-166

26 Grund, C.E., Bahrman, M.P., Balu, N., et al.: 'Dynamic performance characteristics of North American HVDC systems for transient and dynamic stability evaluations', IEEE Trans. Power Appar. Syst., 1981, PAS-100, (7), pp. 3356-3364

27 'IEEE guide for planning DC links terminating at AC locations having low short-circuit capacities', IEEE Std 1204-1997, 1997, p. i

28 'OMIE - Operador del Mercado Ibérico de España.' [Online]. Available: http ://www.omie.es. [Accessed: 29-Jan-2015]

29 Hakim, L., Kubokawa, J., Yuan, Y., et al.: 'A study on the effect of generation shedding to total transfer capability by means of transient stability constrained optimal power flow', IEEE Trans. Power Syst., 2009, 24, (1), pp. 347-355

30 Li, Y.H., Yuan, W.P., Chan, K.W., Liu, M.B.: 'Coordinated preventive control of transient stability with multi-contingency in power systems using trajectory sensitivities', Int. J. Electr. Power Energy Syst., 2011, 33, (1), pp. 147-153

\section{Appendix}

See: Tables 4 and 5

All of the parameters in the following tables have a common 100 MVA base and corresponding voltage rating.

Table 5 Economic data

\begin{tabular}{lc}
\hline Generator & $a$, M.U./MW \\
\hline$G_{1}$ & 70.00 \\
$G_{2}$ & 80.00 \\
$G_{3}$ & 40.00 \\
$G_{4}$ & 100.00 \\
$G_{5}$ & 120.00 \\
HVDC & 55.00 (peak) \\
& 35.00 (off-peak)
\end{tabular}

Table 4 Dynamic parameters of the generators (magnitudes in p.u. referred to base power $100 \mathrm{MVA}$ )

\begin{tabular}{|c|c|c|c|c|c|c|c|c|c|c|}
\hline Generator & $S_{b}, \mathrm{MVA}$ & $R_{\mathrm{a}}$, p.u. & $X_{d}$, p.u. & $X_{d}^{\prime}$, p.u. & $X_{q}$, p.u. & $X_{q}^{\prime}$, p.u. & $H, \mathrm{~s}$ & $D$, p.u. & $T_{d}, \mathrm{~s}$ & $T_{q}, \mathrm{~s}$ \\
\hline $\mathrm{G}_{1}$ & 660 & 0.00 & 0.227 & 0.046 & 0.227 & 0.046 & 21.12 & 13.20 & 39.60 & 6.60 \\
\hline $\mathrm{G}_{2}$ & 520 & 0.00 & 0.290 & 0.058 & 0.290 & 0.058 & 15.51 & 10.34 & 31.02 & 5.17 \\
\hline $\mathrm{G}_{4}$ & 300 & 0.00 & 0.496 & 0.099 & 0.496 & 0.099 & 6.050 & 6.05 & 18.15 & 3.03 \\
\hline $\mathrm{G}_{5}$ & 385 & 0.00 & 0.390 & 0.078 & 0.390 & 0.078 & 7.700 & 7.70 & 23.10 & 3.85 \\
\hline
\end{tabular}

